\title{
Evolution of Conducting Channels in Metallic Atomic Contacts under Elastic Deformation
}

\author{
J. C. Cuevas, ${ }^{1}$ A. Levy Yeyati, ${ }^{1,3}$ A. Martín-Rodero, ${ }^{1}$ G. Rubio Bollinger, ${ }^{2,3}$ C. Untiedt, ${ }^{2}$ and N. Agraït ${ }^{2,3}$ \\ ${ }^{1}$ Departamento de Física Teórica de la Materia Condensada C-V, Universidad Autónoma de Madrid, E-28049 Madrid, Spain \\ ${ }^{2}$ Laboratorio de Bajas Temperaturas, Departamento de Física de la Materia Condensada C-III, Universidad Autónoma de Madrid, \\ E-28049 Madrid, Spain \\ ${ }^{3}$ Instituto Universitario de Ciencia de Materiales "Nicolás Cabrera", Universidad Autónoma de Madrid, E-28049 Madrid, Spain
}

(Received 30 April 1998)

\begin{abstract}
We investigate both experimentally and theoretically the evolution of conductance in metallic one-atom contacts under elastic deformation. While simple metals like Au exhibit almost constant conductance plateaus, $\mathrm{Al}$ and $\mathrm{Pb}$ show inclined plateaus with positive and negative slopes. It is shown how these behaviors can be understood in terms of the orbital structure of the atoms forming the contact. This analysis provides further insight into the issue of conductance quantization in metallic contacts revealing important aspects of their atomic and electronic structures. [S0031-9007(98)07271-8]
\end{abstract}

PACS numbers: 73.40.Jn, 73.20.Dx, 73.40.Cg

In recent experiments a combination of scanning tunneling microscopy (STM) and atomic force microscopy was used to study simultaneously the electrical and mechanical properties of atomic-sized contacts [1]. In these experiments a metallic contact formed between tip and substrate is elongated or contracted. This deformation proceeds in a sequence of elastic stages alternating with sudden atomic rearrangements [2]. During the elastic stages the conductance changes gradually resulting in gently sloped plateaus while the atomic rearrangements show as sharp conductance steps which are hysteretical. The conductance of the last plateau before contact rupture is of the order of one quantum of conductance, $G_{0}=2 e^{2} / h$. This value is typically observed in most experiments on nanocontacts using STM or mechanically controlled break junctions [3] and is generally associated with a one-atom contact [4].

The observed dependence of the conductance on elastic deformation varies from contact to contact but is characteristic for a given metal. In Fig. 1 we show results for three different metals: in the case of $\mathrm{Au}$, the plateaus are rather flat, specially the last one with a nearly constant conductance $\sim G_{0}$; in the case of $\mathrm{Al}$, the conductance increases as the contact is stretched varying typically in the last plateau between $0.5 G_{0}$ and $1 G_{0}$, while for $\mathrm{Pb}$ this variation is in the opposite direction and within the range $3 G_{0}$ to $1 G_{0}$. As will be shown below, these behaviors reflect important aspects of the contact electronic and atomic structures. These experiments were conducted with a STM at low temperatures ( $4.2 \mathrm{~K}$ for $\mathrm{Au}$ and $\mathrm{Al}$ and $1.5 \mathrm{~K}$ for $\mathrm{Pb}$ ) [5]. For the experiments with $\mathrm{Al}$ and $\mathrm{Au}$, the conductance was obtained by measuring the current at a fixed bias voltage of $10 \mathrm{mV}$. In the case of $\mathrm{Pb}$, since it was in the superconducting state, the conductance is given by the high-voltage differential conductance [6].

In the low conductance plateaus the current is carried by a reduced number of conducting channels. While usual transport experiments yield information only on the total conductance, a new technique introduced by Scheer et al. [7] and based on special properties of the superconducting state allows for the determination of the individual channel transmissions. For the specific case of $\mathrm{Al}$, it has been shown that although the total conductance of a one-atom contact is close to one quantum of conductance, there are three channels with non-negligible transmission [7]. In Ref. [8] a microscopic model based on atomic orbitals was introduced which accounts for the number and nature of conducting channels in metallic one-atom contacts.

In this Letter we analyze within this theoretical framework the observed evolution of the last conductance

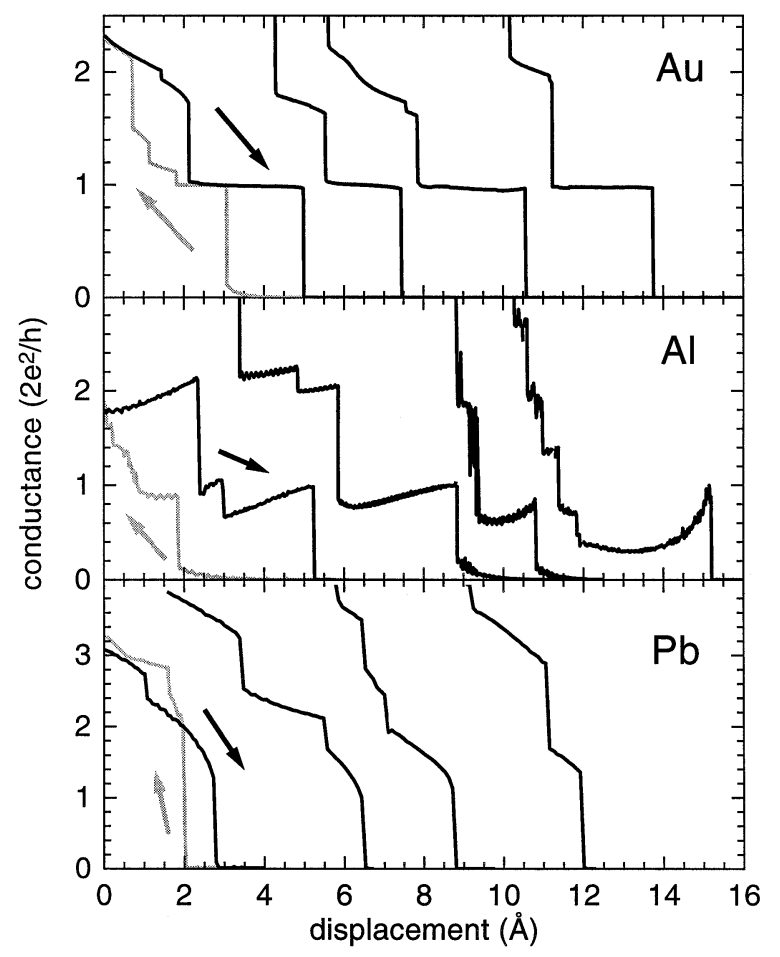

FIG. 1. Evolution of conductance vs tip-sample relative displacement for several representative nanocontacts of $\mathrm{Au}, \mathrm{Al}$, and $\mathrm{Pb}$ in STM experiments. The black and grey curves correspond to elongation and contraction, respectively. 
plateau. We shall show that the characteristic behavior for each metal can be naturally understood in terms of its different atomic orbital structure.

The method is based on a tight-binding model Hamiltonian for the atomic-size contact of the form

$$
\hat{H}=\sum_{i \alpha, \sigma} \epsilon_{i \alpha} c_{i \alpha \sigma}^{\dagger} c_{i \alpha \sigma}+\sum_{i \alpha \neq j \beta, \sigma} t_{i \alpha, j \beta} c_{i \alpha, \sigma}^{\dagger} c_{j \beta, \sigma} .
$$

In this Hamiltonian $i, j$ denote the atomic sites on the contact. We shall consider different model geometries which can be grown from a central atom along a given crystallographic axis on an fcc lattice. After a certain number of layers from the central atom, $N$, this structure is connected to perfect fcc semi-infinite crystals acting as metallic electrodes. Labels $\alpha$ and $\beta$ in Hamiltonian (1) denote the different atomic orbitals at each site. Having a good description of the bulk bands at the Fermi level requires the inclusion of $s$ and $p$ orbitals for metals like $\mathrm{Au}, \mathrm{Al}$, and $\mathrm{Pb}$ (in the case of $\mathrm{Al}$ the effect of including the empty $3 d$ levels will also be discussed). The hopping elements $t_{i \alpha, j \beta}$ and the diagonal elements $\epsilon_{i \alpha}$ are parametrized within a Slater-Koster two center approximation according to Ref. [9] which fairly reproduces the band structure of the bulk material. This parametrization includes a prescription on the variation of the hopping elements with the interatomic distances. In a metallic constriction it is important to determine the electronic charge distribution in a self-consistent way by imposing local charge neutrality [8].

The conductance $G$ can then be calculated in terms of the microscopic parameters using Green function techniques. An expression can be found in which $G$ adopts the Landauer form $G=G_{0} \operatorname{Tr}\left[\hat{t}\left(E_{F}\right) \hat{t}^{\dagger}\left(E_{F}\right)\right]$, where the transmission amplitudes $\hat{t}(E)$ are directly related to the retarded Green function matrix elements (for details, see Ref. [8]). The eigenvalues of the Hermitian matrix $\hat{t} \hat{t}^{\dagger}$ provide the transmission coefficients $\tau_{i}$ of the different conducting channels. Within the present formalism the number of conducting channels with non-negligible transmission for a one-atom contact cannot exceed the number of valence orbitals at the central atom. The actual number is generally smaller, the predictions of the model being in good agreement with recent experimental results for different metallic contacts [6].

We now discuss how the effect of an elastic deformation can be studied within this formalism. A detailed description of the evolution of the ideal constriction geometry with a tensile stress requires knowledge of the force constants between every pair of atoms. However, when analyzing the elastic deformation on the last plateau it is reasonable to assume that the most important effect is due to the weakening of the coupling of the central atom. As shown below, this simple modelization is sufficient to account for the observed tendencies. The distance dependence of the hopping elements is well described by power laws provided that deformations are not larger than 5\%$10 \%$ of the bulk interatomic distances [9].
Figure 2 summarizes the results obtained for the evolution of the total conductance and its decomposition into individual channel contributions for the three metals considered. These results have been obtained for a short neck model geometry $(N=1)$ of a single atom between two perfect semi-infinite crystals grown along the (111) direction, hereafter denoted as the $z$ direction. The results for longer necks $(N>1)$ are qualitatively similar. Notice that both the approximate values of the total conductance as well as their variation upon elastic deformation are in good agreement with the experimental results. This quantitative agreement is encouraging as far as it has proven to be difficult to obtain from $a b$ initio calculations [10].

One can get a deeper insight into the observed trends by analyzing the local density of states (LDOS) at the central atom and the transmissions as a function of energy for each individual channel. In the case of $\mathrm{Au}$ the calculation predicts the presence of a single relevant channel at the Fermi energy [11]; this channel arises essentially from the contribution of the $6 s$ orbitals. As can be observed in the top panel of Fig. 3, the LDOS at the central atom and the transmission exhibit a resonance around the Fermi energy. In this case the charge neutrality condition pins the Fermi energy at the center of the transmission resonance. This provides a strong mechanism accounting for the almost perfect conductance quantization for $\mathrm{Au}$ at the first plateau [12]. The effect of elastic deformations

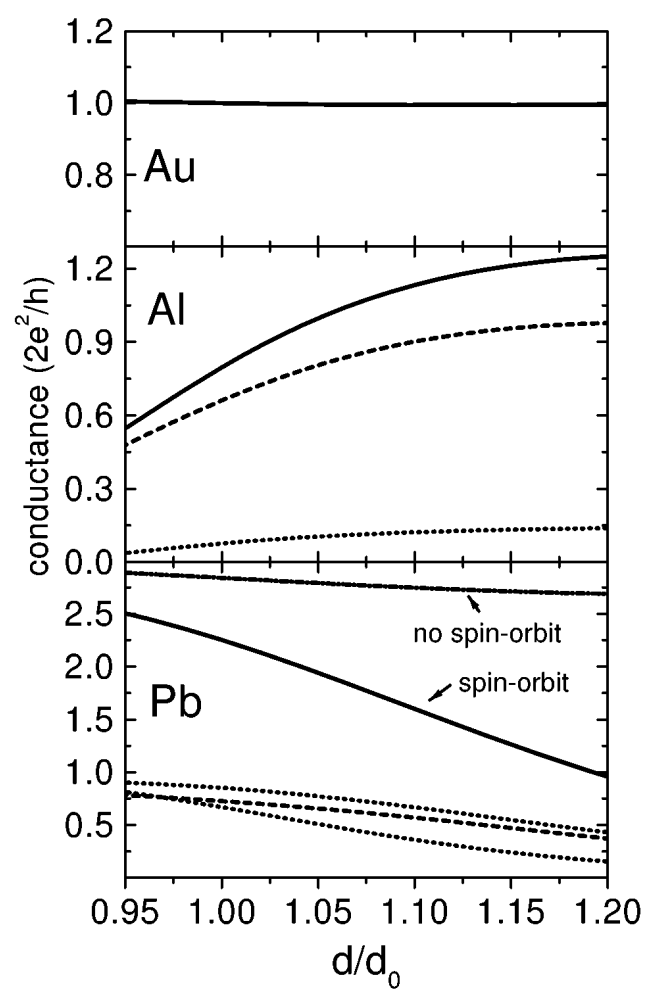

FIG. 2. Calculated last plateau conductance (full line) and its decomposition into eigenchannel contributions for $\mathrm{Au}, \mathrm{Al}$, and $\mathrm{Pb}$ as a function of the distance between the central atom and its first neighbors $d$ in units of the equilibrium distance $d_{0}$. 

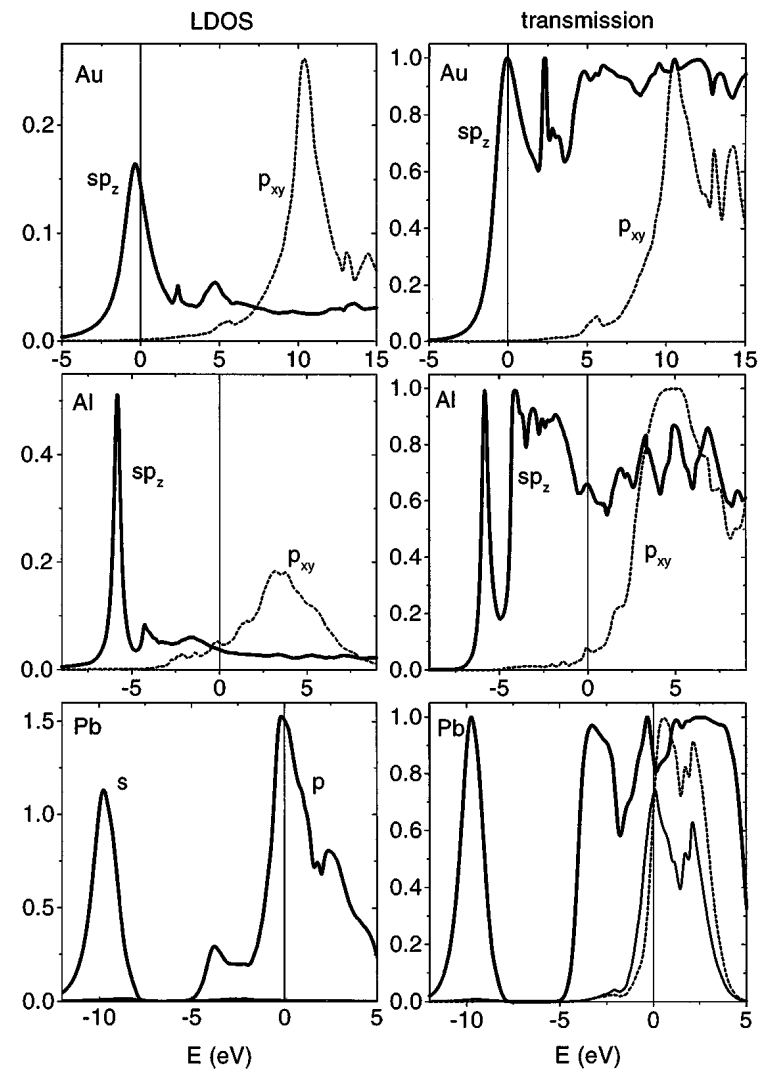

FIG. 3. LDOS at the central atom and channel transmissions vs energy for the ideal geometries $\left(d=d_{0}\right)$ in $\mathrm{Au}, \mathrm{Al}$, and $\mathrm{Pb}$. In the case of $\mathrm{Pb}$ only the total $s$ and $p$ LDOS are shown. The character of the eigenchannels in this case is neither $s p_{z}$ nor $p_{x, y}$ due to spin-orbit coupling. The vertical line indicates the position of the Fermi level.

around this situation is to either broaden or narrow the resonance at the Fermi energy without modifying the value of the total conductance (see upper panel of Fig. 4).

For $\mathrm{Al}$ both $3 s$ and $3 p$ orbitals have an important weight at the Fermi energy. Although the relative position and shape of the $s$ and $p$ bands are similar to the case of $\mathrm{Au}$, the Fermi level lies closer to the center of the $p$ bands. One thus finds three channels with non-negligible transmission: a widely open channel with $s p_{z}$ character and two less transmissive ones with a $p_{x}, p_{y}$ character [8]. This splitting between $p_{z}$ and $p_{x y}$ bands (which are degenerate in the bulk) is due to the neck geometry which shifts the $s p_{z}$ band into lower energies with respect to the center of the $p_{x, y}$ bands (see middle panel in Fig. 3). The $3 d$ orbitals also included in the present calculation do not modify these conclusions but slightly reduce the value of the total transmission. This effect is due to the hybridization between the $p$ and $d$ bands which somewhat shift the $p$ bands to higher energies. In the case of $\mathrm{Al}$, the bond weakening induced by stretching produces a narrowing of the $s p_{z}$ and $p_{x, y}$ bands. This narrowing is more pronounced for the $p_{x, y}$ band and as a consequence the Fermi level tends to lie around the center of the $s p_{z}$ transmission resonance. In the extreme case of a large
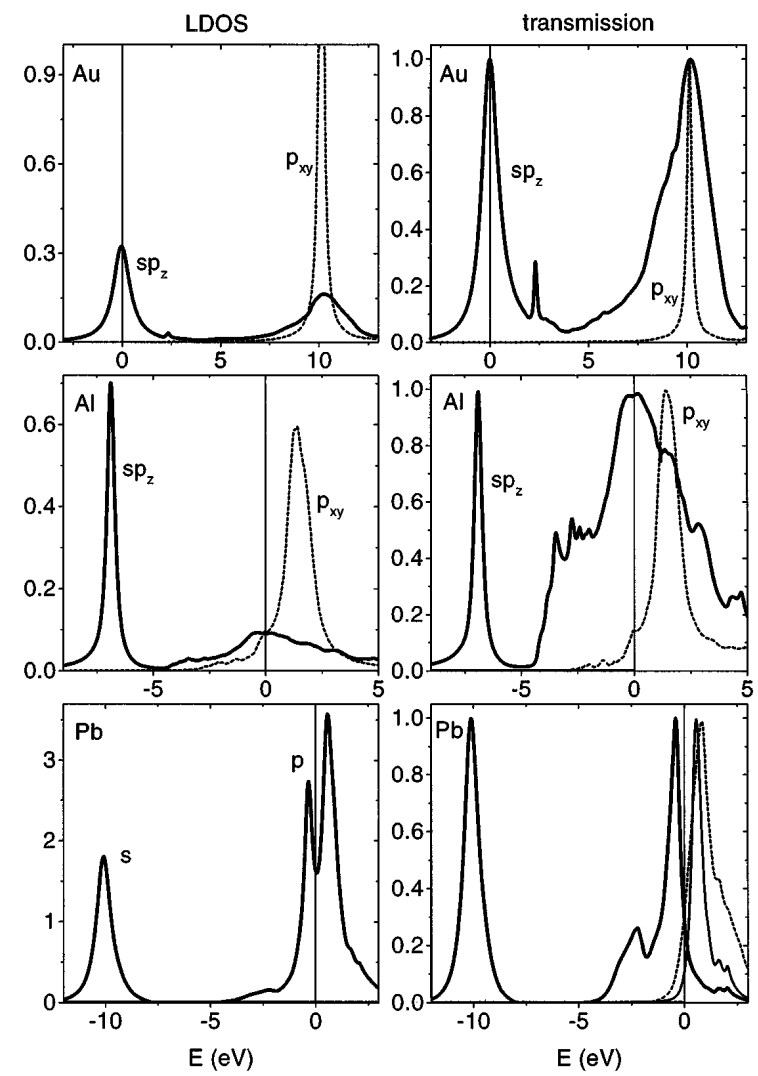

FIG. 4. Same as Fig. 3 for a stretched contact $\left(d=1.2 d_{0}\right)$.

stretching the situation would be similar to that of Au with a single $s p_{z}$ channel with almost perfect transmission (see middle panel of Fig. 4).

The $s$ and $p$ orbitals also play the most important role in the case of $\mathrm{Pb}$ and the number of conducting channels is the same as for $\mathrm{Al}$. However, $\mathrm{Pb}$ has an extra valence electron which moves the Fermi level to an energy region where both $s p_{z}$ and $p_{x, y}$ channels are widely open. The calculated total conductance for the ideal geometry is $\sim 2.8 G_{0}$. As can be observed in the lower panel of Fig. 2, the conductance decrease with elastic elongation is very small in comparison with experimental findings (see Fig. 1). A much better agreement can be obtained by including the effect of spin-orbit coupling in the model calculations. This effect is known to play an important role in the adequate description of the $\mathrm{Pb}$ bulk band structure [13]. Within an atomic orbital basis, the spinorbit coupling leads to an extra term in the Hamiltonian of the form

$$
\hat{H}=\lambda_{\text {so }} \sum_{i, \alpha \beta, \sigma \sigma^{\prime}}\left\langle\alpha \sigma|\vec{L} \vec{S}| \beta \sigma^{\prime}\right\rangle c_{i \alpha \sigma}^{\dagger} c_{i \beta \sigma^{\prime}},
$$

where $\alpha, \beta$ refer to $p$ orbitals at the same lattice site with spin $\sigma, \sigma^{\prime}$, respectively. Typical values of $\lambda_{\text {so }}$ for $\mathrm{Pb}$ are of the order of $0.6 \mathrm{eV}$ [13]. This large coupling produces a splitting of the atomic $p$ levels into $p_{1 / 2}$ and $p_{3 / 2}$ which are separated by $3 \lambda_{\mathrm{so}} / 2$. The evolution of the total 


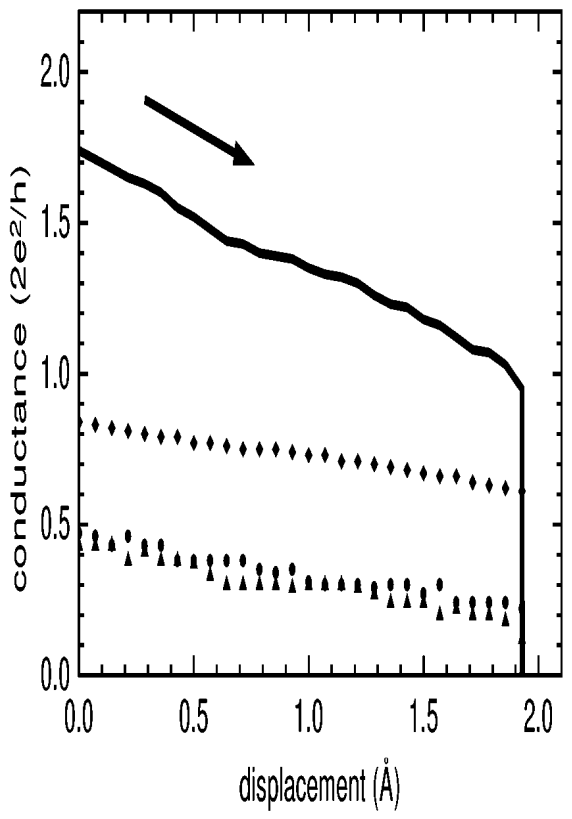

FIG. 5. Experimental conductance for $\mathrm{Pb}$ and its decomposition into three different channels (represented as diamonds, dots, and triangles).

conductance and its decomposition into eigenchannels including spin-orbit coupling is shown in Fig. 2 (lower panel). One can take advantage of the fact that $\mathrm{Pb}$ is in the superconducting state at the temperature at which the experiments were performed $(1.5 \mathrm{~K})$ to obtain the decomposition of the experimental conductance into eigenchannels using the method of Ref. [7]. Figure 5 shows a typical example of the evolution of the decomposition for $\mathrm{Pb}$. The pronounced decrease of the conductance can be understood by analyzing the LDOS at the central atom. For the ideal geometry (Fig. 3 lower panel) the Fermi level lies in the middle of the $p$ band which does not exhibit any structure associated with the splitting of the atomic levels. However, the decomposition into $s p_{z}$ and $p_{x y}$ channels is no longer valid due to the spin-orbit coupling which also breaks the $p_{x y}$ degeneracy. In the elongated contact (Fig. 4 lower panel) the splitting of atomic levels manifests itself in the appearance of a double peaked structure at the $p$ LDOS having a $p_{1 / 2}$ and a $p_{3 / 2}$ character. The Fermi level lies between these two peaks in order to approximately accommodate two electrons in the $p_{1 / 2}$ band. The double peaked structure is also present in the transmission as a function of energy explaining its pronounced decrease with elongation.

The theoretical results presented so far have been obtained for a close packed contact model grown along the (111) direction. This seems the natural choice since (111) faces are known to be energetically favored in fcc metals. We have nevertheless also studied model geometries grown along other crystallographic directions like (100). The number of channels and the approximate values of the total conductance are not dependent on the geometry choice. However, for the particular case of Al one finds a decrease in conductance upon stretching for the (100) case [14], at variance with the experimentally observed tendency.

In summary, we have shown that the conducting channels in metallic one-atom contacts are in general neither completely open nor completely closed, which reflects in the variations of the conductance with strain. Conductance increases or decreases with strain depending on the electronic structure of the element.

The correct description of these transport properties requires going beyond the free-electron approximation. A good semiquantitative agreement with experiments has been achieved using a tight-binding model Hamiltonian based on fittings to the bulk valence bands and a close packed model geometry with the addition of the charge neutrality condition. No special treatment is necessary for each metal indicating the power of the approach.

The authors thank F. Flores and S. Vieira for discussions and continuous support. This work has been supported by the Spanish CICyT under Contracts No. PB93-0260 and No. MAT95-1542.

[1] N. Agraït, G. Rubio, and S. Vieira, Phys. Rev. Lett. 74, 3995 (1995); G. Rubio, N. Agraït, and S. Vieira, Phys. Rev. Lett. 76, 2302 (1996).

[2] U. Landman et al., Science 248, 454 (1990); A. P. Sutton and J. B. Pethica, J. Phys. Condens. Matter 2, 5317 (1990).

[3] For a recent review, see J.M. van Ruitenbeek, in Mesoscopic Electron Transport, edited by L.L. Sohn, L. P. Kouwenhoven, and G. Schön, NATO ASI, Ser. E, Vol. 345 (Kluwer Academic Publishers, Dordrecht, The Netherlands, 1997), p. 549.

[4] N. Agraït et al., Phys. Rev. B 47, 12345 (1993); J. M. Krans et al., Phys. Rev. B 48, 14721 (1993).

[5] For a description of the experimental procedure, see C. Untiedt et al., Phys. Rev. B 56, 2154 (1997).

[6] E. Scheer et al., Nature (London) 394, 154 (1998).

[7] E. Scheer et al., Phys. Rev. Lett. 78, 3535 (1997).

[8] J.C. Cuevas, A. Levy Yeyati, and A. Martín-Rodero, Phys. Rev. Lett. 80, 1066 (1998).

[9] D. A. Papaconstantopoulos, Handbook of the Band Structure of Elemental Solids (Plenum Press, New York, 1986).

[10] Ab initio calculations for one $\mathrm{Al}$ atom between two semiinfinite jelliumlike metals have been presented by N.D. Lang, Phys. Rev. B 52, 5335 (1995); C. C. Wan et al., Appl. Phys. Lett. 71, 419 (1997).

[11] Similar findings using a local density approximation for $\mathrm{Au}$ were reported by M. Brandbyge, M. R. Sorensen, and K. W. Jacobsen, Phys. Rev. B 56, 14956 (1997).

[12] A. Levy Yeyati, A. Martín-Rodero, and F. Flores, Phys. Rev. B 56, 10369 (1997).

[13] K. Würde, A. Mazur, and J. Pollmann, Phys. Rev. B 49, 7679 (1994).

[14] This opposite behavior for (111) and (100) directions has also been found for the conductivity of bulk Al. See D. Sánchez-Portal et al., Phys. Rev. Lett. 79, 4198 (1997). 